\title{
684.
}

\section{ON A RELATION BETWEEN CERTAIN PRODUCTS OF DIFFERENCES.}

[From the Quarterly Journal of Pure and Applied Mathematics, vol. xv. (1878), pp. 174, 175.]

CoNSIDER the function

where

$$
\left\{\begin{array}{r}
a b c \cdot d e \\
+b c d \cdot e a \\
+c d e \cdot a b \\
+d e a \cdot b c \\
+e a b \cdot c d
\end{array}\right\} \quad\left\{\begin{array}{r}
a b d \cdot c e \\
+b c e \cdot d a \\
+c d a \cdot e b \\
+d e b \cdot a c \\
+e a c \cdot b d
\end{array}\right\}
$$

$$
\begin{aligned}
& a b c=(a-b)(b-c)(c-a), \\
& a b=(a-b)(b-a),=-(a-b)^{2} \\
& \& c .
\end{aligned}
$$

therefore

$$
\begin{aligned}
& a b c=b c a=c a b=-b a c, \& c . ; \\
& a b=b a .
\end{aligned}
$$

It is to be shown that the function vanishes if $e=d$. Writing $e=d$, the value is

$$
\begin{aligned}
3(b c d \cdot d a+d a b \cdot c d) & -a b d \cdot c d \\
& -b c d \cdot d a \\
& -c d a \cdot d b \\
& -d a c \cdot b d
\end{aligned}
$$


viz. this is

$$
\begin{aligned}
& 3 b c d \cdot a d-a b d \cdot c d \\
+ & 3 a b d \cdot c d-b c d \cdot a d \\
& -2 a c d \cdot b d \\
= & 2 b c d \cdot a d-2 a c d \cdot b d+2 a b d \cdot c d \\
= & 2(b c d \cdot a d+c a d \cdot b d+a b d \cdot c d)
\end{aligned}
$$

which is easily seen to vanish; the value is

$$
\begin{aligned}
& (b-c)(c-d)(d-b)(a-d)^{2}=-(b-c)(a-d)^{2}(b-d)(c-d) \\
+ & (c-a)(a-d)(d-c)(b-d)^{2}-(c-a)(a-d)(b-d)^{2}(c-d) \\
+ & (a-b)(b-d)(d-a)(c-d)^{2}-(a-b)(a-d)(b-d)(c-d)^{2}:
\end{aligned}
$$

viz. omitting the factor $(a-d)(b-d)(c-d)$, this is

$$
\begin{aligned}
= & -(b-c)(a-d) \\
& -(c-a)(b-d) \\
& -(a-b)(c-d),
\end{aligned}
$$

which vanishes. Hence the function also vanishes if $e=a$, or $a=b$ or $b=c$, or $c=d$; and it is thus a mere numerical multiple of $(a-b)(b-c)(c-d)(d-e)(e-a)$, or say it is $=$ Mabcde.

To find $M$ write $e=c$, the equation becomes

$$
\begin{aligned}
& 3 a b c \cdot d c-c d a \cdot c b=M a b c d c,=M a b c \cdot d c \\
+ & 3 b c d \cdot c a-a c \\
+ & 3 d c a \cdot b c \\
+ & 3 c a b \cdot c d
\end{aligned}
$$

viz. this is

$$
6 a b c \cdot d c+4 d b c \cdot a c+4 a d c \cdot b c=M \cdot a b c \cdot d c,
$$

giving $M=10$. In fact, we then have

that is,

$$
-4 a b c \cdot d c+4 d b c \cdot a c+4 a d c \cdot b c=0,
$$

$$
-a b c \cdot d c-b d c \cdot a c-d a c \cdot b c=0,
$$

which is right. And we have thus the identity

or say

$$
\left\{\begin{array}{r}
a b c \cdot d e \\
+b c d \cdot e a \\
+c d e \cdot a b \\
+d e a \cdot b c \\
+e a b \cdot c d
\end{array}\right\} \quad\left\{\begin{array}{r}
a b d \cdot c e \\
+b c e \cdot d a \\
+c d a \cdot e b \\
+d e b \cdot a c \\
+e a c \cdot b d
\end{array}\right\}=10 \cdot a b c d e
$$

$$
3[a b c d e]-[a c e b d]=10\{a b c d e\} .
$$

